\title{
Evaluation of three commercial detection systems for Mycobacterium tuberculosis where clinical diagnosis is difficult
}

\author{
T J Brown, E G M Power, G L French
}

\begin{abstract}
Aims-To assess the performance of three commercially available Mycobacterium tuberculosis detection systems employing nucleic acid amplification, when applied directly to respiratory and nonrespiratory specimens from patients where the diagnosis of tuberculosis is difficult using clinical and traditional bacteriological methods.
\end{abstract}

Methods-42 respiratory and 21 nonrespiratory specimens were concentrated, examined with auramine staining, and cultured on Lowenstein-Jensen slopes. These specimens were also assayed using the Amplicor Mycobacterium tuberculosis test (AM) (Roche Diagnostic Systems), the Amplified Mycobacterium tuberculosis direct test (AMD) (Gen-Probe), and the LCx Mycobacterium tuberculosis assay (LMA) (Abbott Laboratories).

Results-All three amplification systems used in this study gave specificities of $100 \%$ when used on respiratory specimens. When used on non-respiratory specimens, AM and LMA gave specificities of $100 \%$ and AMD $75 \%$. With respiratory specimens the AM, AMD, and LMA systems gave sensitivities of $75 \%, 65.2 \%$, and $79.2 \%$, respectively. With nonrespiratory specimens the sensitivities were $50 \%, 66.7 \%$, and $60 \%$, while with smear negative, culture positive specimens they were $33.3 \%, 66.7 \%$, and $55.6 \%$. Positive predictive values of $100 \%$ were seen with all specimens except nonrespiratory specimens assayed using AMD where the value was $66.7 \%$.

Conclusions-The manufacturers of these systems recommend that they should only be used for the direct analysis of respiratory specimens, and the US Food and Drug Administration has approved them for use only with smear positive specimens. This study confirms that sensitivities are lower for non-respiratory and smear negative specimens, but positive predictive values are high. Provided they are interpreted with caution, positive results with these tests in respiratory and non-respiratory specimens are useful in tuberculous patients who are otherwise difficult to diagnose. Each amplification has advantages and disadvantages compared with the others. (f Clin Pathol 1999;52:193-197)
Keywords: Mycobacterium tuberculosis; polymerase chain reaction; nucleic acid amplification; diagnosis

The majority of cases of uncomplicated pulmonary tuberculosis are initially diagnosed on the basis of the clinical presentation and by the presence of acid fast bacilli (AFB) in sputum specimens. Treatment is started and the diagnosis confirmed two to six weeks later by culture results. However, where the infection is non-pulmonary, when the clinical presentation is atypical, or when infection is with mycobacteria other than Mycobacterium tuberculosis (MOTT), the diagnosis is more difficult. In these cases clinical management is more dependent on the results of laboratory investigation.

Molecular methods of diagnosis, especially those involving nucleic acid amplification, are being increasingly used for the diagnosis of mycobacterial infection, both for the identification of mycobacterial isolates ${ }^{1}$ and for the detection of mycobacteria in clinical specimens. $^{2}$ Several commercial systems of nucleic acid amplification are now available for the detection of $M$ tuberculosis in clinical laboratories. The manufacturers recommend that they should be used only for respiratory specimens, and the US American Food and Drug Administration has so far only approved their use with smear positive respiratory specimens. ${ }^{3}$ The ease of introduction and the use of standardised reagents make the use of commercially available $M$ tuberculosis detection systems attractive to non-specialised laboratories

The rapid confirmation of $M$ tuberculosis in cases of uncomplicated, sputum smear positive pulmonary tuberculosis may be useful but such results have little impact on the clinical management of these cases. In immunocompromised patients, however, the rapid differentiation between pulmonary infection with $M$ tuberculosis and MOTT is important for both treatment and infection control, for example in patients with AIDS. $M$ tuberculosis and MOTT are isolated from both respiratory and nonrespiratory specimens from the patient population used in this study. Rapid methods for distinguishing $M$ tuberculosis and MOTT in both respiratory and non-respiratory specimens would thus be useful. These nucleic acid amplification systems would also make a significant contribution to the clinical management of tuberculous patients with atypical presentations if they could be applied to microscopy smear negative or extrapulmonary
Accepted for publication 9 November 1998. 
specimens. In this study we investigated commercial methods for the detection of mycobacterial DNA in these critical specimens.

We used three commercial systems for the detection of $M$ tuberculosis nucleic acid: the Amplicor Mycobacterium tuberculosis test (Roche Diagnostic Systems), the Amplified Mycobacterium tuberculosis direct test (GenProbe), and the LCx Mycobacterium tuberculosis assay (Abbott Laboratories). We applied these to specimens from any site in which AFB were seen on microscopy, to all specimens that were AFB negative on microscopy but which yielded $M$ tuberculosis on culture, and to other specimens where the clinical diagnosis of tuberculosis was difficult and clinicians had therefore specifically asked for nucleic acid amplification tests.

\section{Methods}

SPECIMENS

These were received in our clinical laboratory with a request for investigation for mycobacteria between August 1996 and January 1997. Sixty three specimens from 48 patients were examined, including 12 sputum specimens known to be negative for $M$ tuberculosis which were used as controls. There were 42 respiratory and 21 non-respiratory specimens. In 34 specimens AFB were seen on microscopy ("microscopy positive") and $M$ tuberculosis was cultured from 21, MOTT from eight, and no mycobacteria from five. In 11 specimens, AFB were not seen on initial microscopy ("microscopy negative") but yielded $M$ tuberculosis on culture ("culture positive"); a further six specimens were from patients with clinical evidence of possible $M$ tuberculosis infection.

SPECIMEN PROCESSING

All specimens were stored at $4^{\circ} \mathrm{C}$ before testing, except for the microscopy negative/ culture positive specimens which were stored at $-20^{\circ} \mathrm{C}$ until the results of the cultures were known.

Respiratory and faecal specimens were decontaminated using $2 \% \mathrm{NaOH}$ and concentrated by centrifugation before being neutralised. Tissues, biopsies, and pus specimens were decontaminated by washing with $6 \%$ sulphuric acid before being neutralised. Pleural fluid was spun at $1700 \mathrm{~g}$ and the supernatant discarded. Pellets from treated specimens were examined for AFB using auramine fluorochrome staining and inoculated onto Lowenstein-Jensen slopes for culture at $37^{\circ} \mathrm{C}$ for up to eight weeks. The remainder of the specimen was stored at $4^{\circ} \mathrm{C}$ for nucleic acid amplification detection of $M$ tuberculosis. Blood and bone marrow were inoculated directly into Bactec13A culture bottles (Becton Dickinson) and no microscopic examination was performed.

For nucleic acid amplification detection of $M$ tuberculosis in these specimens, up to $1.5 \mathrm{ml}$ of heparinised blood or $100 \mu \mathrm{l}$ of bone marrow were centrifuged at $12500 \mathrm{~g}$ for 10 minutes; the supernatant was aspirated and $1 \mathrm{ml}$ of distilled water was added to the pellet; the tube was vortexed and the tube recentrifuged; this was repeated until the supernatant was clear; the pellet was then stored as above.

NUCLEIC ACID AMPLIFICATION

The three commercial systems examined in this study were:

(1) The Amplicor Mycobacterium tuberculosis test (Roche Diagnostic Systems) - A region of the gene encoding the $16 \mathrm{~S}$ ribosomal subunit (4) is amplified by the polymerase chain reaction (PCR) with the incorporation of a biotin label; the labelled amplicon is hybridised to a complementary $M$ tuberculosis specific probe immobilised in a microtitre tray; hybridisation is detected by a colorimetric assay following a streptavidin-alkaline phosphatase conjugate reaction.

(2) The Amplified Mycobacterium tuberculosis direct test (Gen-Probe)-A portion of $\mathrm{rRNA}^{4}$ is amplified by transcription mediated amplification; the resulting amplification products are probed with a $M$ tuberculosis specific probe labelled with acridinium ester; hybridisation is detected by a luminescent hybridisation protection assay. ${ }^{5}$

(3) The LCx Mycobacterium tuberculosis assay (Abbott Laboratories)-A portion of the gene encoding protein antigen $b^{6}$ is amplified by the ligase chain reaction (LCR). ${ }^{7}$ The oligonucleotide primers are labelled so that the resulting amplification products have a capture hapten at one terminus and a detection hapten at the other. The LCR product is detected by a microbead enzyme immunoassay using alkaline phosphatase and fluorimetry. ${ }^{8}$

Amplifications were carried out according to the manufacturers' instructions provided with the kits. ${ }^{9-11}$

ANALYSIS OF RESULTS

The total number of true positive specimens was taken as the number of $M$ tuberculosis culture positive specimens plus microscopy positive, culture negative specimens with strong clinical evidence of $M$ tuberculosis infection. Percentage sensitivity, specificity, positive predictive value (PPV), and negative predictive value (NPV) were calculated as follows:

Sensitivity $=$ detected positive/true positive $\times$ 100

Specificity $=$ detected negative/true negative $\times$ 100

$\mathrm{PPV}=$ detected true positive/detected positive $\times 100$

$\mathrm{NPV}=$ detected true negative/detected negative $\times 100$

\section{Results}

Of the 63 specimens examined in this study, $47.6 \%$ were positive for $M$ tuberculosis and $15.9 \%$ for MOTT. These were selected from a laboratory where the positivity rate of all specimens examined was $5.4 \%$ for $M$ tuberculosis and $2.0 \%$ for MOTT, as seen in table 1 .

The specimen types, smear results, culture results, and amplification assay results are summarised in table 2. Auramine staining can 
Table 1 Background positivity rate in specimens received for mycobacterial investigation at St Thomas' Hospital, London, August 1995 to August 1997

\begin{tabular}{|c|c|c|c|}
\hline & $\begin{array}{l}\text { Per cent } \\
\text { culture+/ } \\
\text { smear+ }\end{array}$ & $\begin{array}{l}\text { Per cent } \\
\text { culture+/ } \\
\text { smear- }\end{array}$ & $\begin{array}{l}\text { Per cent } \\
\text { total } \\
\text { culture+ }\end{array}$ \\
\hline \multicolumn{4}{|c|}{ Respiratory specimens $(n=4826)$ : } \\
\hline $\begin{array}{l}\text { Mtb } \\
(\text { MOTT) }\end{array}$ & $\begin{array}{l}3.2 \\
(0.4)\end{array}$ & $\begin{array}{l}1.1 \\
(1.2)\end{array}$ & $\begin{array}{l}4.3 \\
(1.2)\end{array}$ \\
\hline \multicolumn{4}{|c|}{$\begin{array}{l}\text { Non-respiratory specimens } \\
\quad(\mathrm{n}=3137) \text { : }\end{array}$} \\
\hline $\begin{array}{l}\text { Mtb } \\
(\text { MOTT) }\end{array}$ & $\begin{array}{l}5.4 \\
(1.6)\end{array}$ & $\begin{array}{l}2.1 \\
(0.8)\end{array}$ & $\begin{array}{l}7.5 \\
(2.4)\end{array}$ \\
\hline \multicolumn{4}{|c|}{ All specimens $(n=7963)$ : } \\
\hline $\begin{array}{l}\text { Mtb } \\
\text { (MOTT) }\end{array}$ & $\begin{array}{l}3.9 \\
(0.9)\end{array}$ & $\begin{array}{l}1.5 \\
(1.1)\end{array}$ & $\begin{array}{l}5.4 \\
(2.0)\end{array}$ \\
\hline
\end{tabular}

MOTT, mycobacteria other than $M$ tuberculosis; $\mathrm{Mtb}$,

Mycobacterium tuberculosis.

produce false positive smear results. In this study all smear positive specimens were also Mycobacterium $s p p$ culture positive or from patients from whom $M$ tuberculosis had been previously cultured.

Smear positive respiratory specimens

The amplification assays are recommended for use on smear positive respiratory specimens. Table 3 shows sensitivity, specificity, PPV, and NPV results from the 24 specimens of this type in the study. $M$ tuberculosis was cultured from 16 of these specimens, giving a PPV of $66.6 \%$ for auramine smears, compared with 100\% with all three commercial amplification systems. The specificity on this group of specimens was $100 \%$ with all three systems. The sensitivity with both the Amplicor Mycobacterium tuberculosis test and the LCx Mycobacterium tuberculosis assay was $84.2 \%$, while with the Amplified Mycobacterium tuberculosis direct test it was $57.9 \%$. Different true positives were missed by the different systems; thus the combined sensitivity of the three tests on these specimens was $94.4 \%$ (table 3 ).

\section{Respiratory and non-respiratory specimens combined}

When both respiratory and non-respiratory specimens were assayed, the specificity of both the Amplicor Mycobacterium tuberculosis test and the LCx Mycobacterium tuberculosis assay was $100 \%$, while it was $92.5 \%$ with the Amplified Mycobacterium tuberculosis direct test and $64.5 \%$ with auramine staining for AFB (table 4). With the non-respiratory specimens, the Amplicor Mycobacterium tuberculosis test and the LCx Mycobacterium tuberculosis assay gave sensitivities of $50 \%$ and $60 \%$, respectively, and
Table 3 Comparison statistics for smear positive respiratory specimens showing positive predictive value $(P P V)$ and negative predictive value (NPV)

\begin{tabular}{lllll}
\hline & $\begin{array}{l}\text { Per cent } \\
\text { sensitivity }\end{array}$ & $\begin{array}{l}\text { Per cent } \\
\text { specificity }\end{array}$ & $\begin{array}{l}\text { Per cent } \\
\text { PPV }\end{array}$ & $\begin{array}{l}\text { Per cent } \\
\text { NPV }\end{array}$ \\
\hline Gen-probe MTD & 57.9 & 100 & 100 & 75.9 \\
Abbott LCx & 84.2 & 100 & 100 & 91.7 \\
$\begin{array}{l}\text { Amplicor } \\
\text { Three systems }\end{array}$ & 84.2 & 100 & 100 & 91.7 \\
$\quad$ combined & 94.4 & 100 & 100 & 95.6 \\
\hline NPV, negative predictive value; & PPV, positive predicitive value.
\end{tabular}

Table 4 Comparison of statistics for all the specimens used in this study showing specificity, sensitivity, positive predictive value (PPV), and negative predictive value (NPV)

\begin{tabular}{lllll}
\hline & $\begin{array}{l}\text { Per cent } \\
\text { sensitivity }\end{array}$ & $\begin{array}{l}\text { Per cent } \\
\text { specificity }\end{array}$ & $\begin{array}{l}\text { Per cent } \\
\text { PPV }\end{array}$ & $\begin{array}{l}\text { Per cent } \\
\text { NPV }\end{array}$ \\
\hline All specimens & & & & \\
$\quad$ Auramine smear & 71.8 & 64.5 & 67.6 & 64.5 \\
Gen-probe MTD & 65.6 & 92.5 & 87.5 & 77.1 \\
Abbott LCx & 75.0 & 100 & 100 & 79.5 \\
$\quad$ Amplicor & 71.8 & 100 & 100 & 77.5 \\
Respiratory specimens & & & & \\
$\quad$ Gen-probe MTD & 65.2 & 100 & 100 & 65.4 \\
Abbott LCx & 79.2 & 100 & 100 & 77.2 \\
$\quad$ Amplicor & 75.0 & 100 & 100 & 74.0 \\
Non-respiratory specimens & & & \\
$\quad$ Gen-probe MTD & 66.7 & 75 & 66.7 & 75 \\
Abbott LCx & 60.0 & 100 & 100 & 76.5 \\
Amplicor & 50.0 & 100 & 100 & 72.2 \\
Hospital requests & & & & \\
$\quad$ Gen-probe MTD & 100 & 75 & 60 & 100 \\
Abbott LCx & 33.3 & 100 & 100 & 20 \\
Amplicor & 100 & 100 & 100 & 100 \\
Smear-/ culture+ & & & & \\
$\quad$ Gen-probe MTD & 66.7 & 100 & 100 & 40 \\
Abbott LCx & 55.6 & 100 & 100 & 33.3 \\
Amplicor & 33.3 & 100 & 100 & 25.0 \\
\end{tabular}

specificity of $100 \%$. The sensitivity for the Amplified Mycobacterium tuberculosis direct test was $66.6 \%$ but the specificity was $75 \%$.

Specimens from patients with uncertain clinical diagnosis

Eleven specimens from patients with an uncertain clinical diagnosis (half of which were AFB microscopy negative) gave sensitivities of $100 \%$ (table 4) with the Amplified Mycobacterium tuberculosis direct test and the Amplicor Mycobacterium tuberculosis test, but only 33.3\% with the LCx Mycobacterium tuberculosis assay (table 4). Specificity was $75 \%$ with the Amplified Mycobacterium tuberculosis direct test but $100 \%$ with the other two systems. The clinical selection process for these specimens raised the positivity rate from the $5.4 \%$ normally seen in specimens submitted for mycobacterial investigations (table 1 ) to $27.3 \%$.

Table 2 Summary of positive results according to specimen type

\begin{tabular}{lllllll}
\hline Specimen type & $\begin{array}{l}\text { Number of } \\
\text { specimens }\end{array}$ & Smear + & Culture + & Amplicor + & Genprobe + & Abbott + \\
\hline Respiratory & 42 & 24 & $21+$ & 18 & 15 & 19 \\
Tissue/biopsy & 5 & 2 & 2 & 2 & 2 & 1 \\
Pus & 6 & 3 & 5 & 3 & 3 & 3 \\
Urine & 4 & 1 & 1 & 0 & 3 & 0 \\
Pleural fluid & 1 & 0 & 1 & 0 & 0 & 0 \\
Faeces & 1 & 1 & 0 & 0 & 1 & 0 \\
Bone marrow/ blood culture & 4 & 3 & 0 & 23 & 24 & 24 \\
Total & 63 & 34 & 30 & 23 & 0 \\
\hline
\end{tabular}

†Excluding two culture negative sputum specimens, one culture positive 30 weeks previously, one culture positive two days previously; 23 M tuberculosis positive specimens were used in the calculations. 
Microscopy negative, culture positive specimens The Amplified Mycobacterium tuberculosis direct test was the most sensitive test for specimens that were microscopy negative and culture positive, with a sensitivity of $66.7 \%$; the LCx Mycobacterium tuberculosis assay and the Amplicor Mycobacterium tuberculosis test had sensitivities of $55.6 \%$ and $33.3 \%$, respectively, with this group. Specificity was $100 \%$ for all the systems with these specimens.

\section{Discussion}

Where the Amplicor Mycobacterium tuberculosis test and the Amplified Mycobacterium tuberculosis direct test have been compared in studies of microscopy positive respiratory specimens, specificities have been reported as being between $94.4 \%$ and $100 \% .{ }^{12-15}$ In these studies between $10.5 \%$ and $34.1 \%$ of the tested specimens were positive for $M$ tuberculosis. The specificity of the LCx Mycobacterium tuberculosis assay has been reported by its manufacturer ${ }^{9}$ to be $99.3 \%$ for 1971 respiratory specimens, of which $8 \%$ were culture positive. Other studies show $100 \%$ specificity in a sample that was $37.5 \%$ culture positive ${ }^{8}$ and $99.2 \%$ in a sample that was $25.5 \%$ culture positive. ${ }^{16}$

Our results confirm that the probes used in commercial $M$ tuberculosis assays are highly specific, although the Amplified Mycobacterium tuberculosis direct test was less so when used on non-respiratory specimens. The specificity of the amplification techniques described here was far higher than that using auramine smears on these specimens, although sensitivity was of the same order. The specificity seen in other studies suggests that the specificity would be reduced if the assays were to be carried out on unselected specimens. However, the purpose of this study was to compare the sensitivities of the commercial assays. Previous studies have reported the sensitivities for both the Amplicor Mycobacterium tuberculosis test and the Amplified Mycobacterium tuberculosis direct test to be between $80 \%$ and $99 \%$ when used to test microscopy positive respiratory specimens. ${ }^{12-15}$ The LCx Mycobacterium tuberculosis assay is claimed by its manufacturers to have a sensitivity of $93.7 \%{ }^{9}$ with similar specimens, and other reports have shown sensitivities between $90.8 \%$ and $90.2 \% .^{816}$

In our present study, the results for the Amplicor Mycobacterium tuberculosis test for specimens that were both microscopy positive and culture positive were similar to those reported by others. ${ }^{12-15}$ However, the sensitivities of the Amplified Mycobacterium tuberculosis direct test and the LCx Mycobacterium tuberculosis assay tests (table $3^{3}$ ) were lower than previously reported. ${ }^{8916}$ In our study, where the three assays failed to amplify target nucleic acid it was not always in the same specimens, and when the results of the three tests were combined for each specimen the sensitivity rose to $94.4 \%$ - a figure at the upper end of the results reported by others. This may have been because multiple assays were performed on each specimen and because of variable inhibition of the different enzymes used in the three assays by substances in the specimens.
The sensitivities seen with Amplicor $\mathrm{Myco-}$ bacterium tuberculosis test and the LCx Mycobacterium tuberculosis assay in non-respiratory specimens were lower than those seen in respiratory specimens. This fall in sensitivity was also reported when a non-commercial PCR assay using IS6110 as target showed a sensitivity of $89 \%$ with respiratory specimens and a reduced sensitivity of $42 \%$ with nonrespiratory specimens. ${ }^{17}$ The Amplified $\mathrm{Myco}-$ bacterium tuberculosis direct test showed approximately the same sensitivity in both respiratory and non-respiratory specimens, although the specificity fell in the latter.

When smear negative, culture positive specimens were examined, the Amplicor Mycobacterium tuberculosis test and LCx Mycobacterium tuberculosis assay showed reductions in sensitivity of the magnitude seen in previous studies, ${ }^{3}{ }^{16}$ when compared with smear positive specimens. The Amplified Mycobacterium tuberculosis direct test showed no such reduction in sensitivity.

The Amplified Mycobacterium tuberculosis direct test should have the highest sensitivity since each target cell may contain rRNA templates at a concentration $10^{3}$ times that of the DNA targets used in the other assays. ${ }^{4}$ In this study the Amplified Mycobacterium tuberculosis direct test was the most sensitive when examining the smear negative but culture positive specimens, a group for which there is no traditional rapid method. As the same amplification failure rate is seen with both smear positive and smear negative specimens, failure of amplification must be caused by factors other than low template copy number-for example problems in specimen preparation.

A cause of false positive results in amplification based tests is amplicon contamination. This risk is usually minimised by separation of the reagent preparation area, the specimen preparation area, and the amplicon detection area. The Amplicor Mycobacterium tuberculosis test further reduces this risk by incorporating uracil rather than thymine in its amplicon. Contaminating amplicon from previous assays can therefore be degraded using uracil DNA glycosylase, ${ }^{18}$ leaving only non-amplicon DNA as the amplification template. The use of an RNA amplicon by the Amplified Mycobacterium tuberculosis direct test may reduce the risk of amplicon contamination when compared with systems using DNA amplicon, as RNA is less stable than DNA. False positives were seen in this study when non-respiratory specimens were assayed using the Amplified Mycobacterium tuberculosis direct test. We noted with interest that if the cut off was raised for these specimens to from 30000 relative light units (rlu) to $150000 \mathrm{rlu}$, no false positives were seen, with the loss of one true positive with a low rlu value. No false positives with either of the other systems were seen in this study.

The amount of bench time, the cost, and the technical expertise required for the three systems is similar. The Amplified Mycobacterium tuberculosis direct test could be carried out in the shortest time (approximately 5.5 hours from reception of the specimen) although all 
three systems could give results in an eight hour working day. The Amplicor Mycobacterium tuberculosis test uses little designated equipment and therefore can be introduced easily into a diagnostic laboratory where PCR techniques are already employed. All the equipment and materials required for the LCx Mycobacterium tuberculosis assay are supplied in the kit, making it the easiest system to use in a non-specialist laboratory, but the large amount of designated equipment to be housed in containment facilities may present problems.

In this study we have shown that these commercial kits can be used to detect $M$ tuberculosis in specimens from clinically difficult cases. Although the sensitivities are lower with non-respiratory or smear negative specimens than with smear positive respiratory specimens, the PPV remains at or near $100 \%$. The three different systems each had some advantages over the others. The LCx Mycobacterium tuberculosis assay showed the highest sensitivity overall and would be the easiest system to use in a non-specialist laboratory as all the equipment and reagents are supplied with the system. However, in smear negative and culture positive specimens, results from which are possibly the most clinically useful, the Amplified Mycobacterium tuberculosis direct test was the most sensitive system. The only system that addresses the problem of amplicon contamination is the Amplicor Mycobacterium tuberculosis test.

1 Lebrun L, Espinasse F, Poveda JD, et al. Evaluation of nonradioactive probes for identification of mycobacteria. $\mathcal{F}$ Clin Microbiol 1992;30:2476-8.

2 Hawkey PM. The role of polymerase chain reaction in the diagnosis of mycobacterial infections. Rev Med Microbiol diagnosis of myct
3 American Thoracic Society Workshop. Rapid diagnostic tests for tuberculosis: what is the appropriate use? $A m \mathcal{F}$ tests for tuberculosis: what is the app
Resp Crit Care Med 1997;155:1804-14.

4 Boddinghaus B, Rogall T, Flohr T, et al. Detection and identification of mycobacteria by amplification of rRNA. $\mathcal{F}$ Clin Microbiol 1990;28:1751-9.

5 Hill CS. Gen-Probe transcription-mediated amplification: system principles. San Diego: Gen-Probe Inc, 1996.

6 Andersen AB, Hansen EB. Structure and mapping of antigenic domains of protein antigen b, a 38,000molecular-weight protein of Mycobacterium tuberculosis. Infect Immun 1989;57:2481-8.

7 Leckie GW, Lee HH. Infectious disease testing by ligase chain reaction. In: Meyers RA, ed. Molecular biology and biotechnology. New York: VCH, 1995:463-6.

8 Ausina V, Gamboa F, Gazapo E, et al. Evaluation of the semiautomated Abbott LCx Mycobacterium Tuberculosis assay for the direct detection of Mycobacterium tuberculosis in respiratory specimens. F Clin Microbiol 1997;35:19962002

9 LCX Mycobacterium tuberculosis assay. Product manual. Abbott Park, IL: Abbott Laboratories, 1996.

10 Gen-Probe amplified Mycobacterium tuberculosis direct test. Product manual. San Diego, California: Gen-Probe Inc, 1995.

11 Amplicor Mycobacterium tuberculosis test. Product manual. Branchburg, NJ: Roche Diagnostic Systems, 1994.

12 Zolnir-Dovc M, Poljak M, Seme K, et al. Evaluation of two commercial amplification assays for the detection of $\mathrm{Myco-}$ bacterium tuberculosis complex in respiratory specimens. Infection 1995;23:216-21.

13 Ichiyama S, Iinuma Y, Tawada Y, et al. Evaluation of Gen-Probe amplified Mycobacterium tuberculosis direct test and Roche PCR-microwell plate hybridization method (Amplicor Mycobacterium) for direct detection of mycobacteria. $\mathcal{F}$ Clin Microbiol 1996;34:130-3.

14 Vuorinen P, Meittinen A, Vuento R, et al. Direct detection of Mycobacterium tuberculosis in respiratory specimens by Gen-Probe amplified Mycobacterium tuberculosis direct test and Roche Amplicor Mycobacterium tuberculosis test. F Clin Microbiol 1995;33:1856-9.

15 Dalovisio JR, Montenegro-James S, Kemmerly SA, et al. Comparison of the Amplified Mycobacterium tuberculosis (MTB) direct test, Amplicor MTB PCR, and IS6110-pcr for detection of MTB in respiratory specimens. Clin Infect Dis 1996;23:1099-106.

16 Lindbrathen A, Gaustad P, Hovig B, et al. Direct detection of Mycobacterium tuberculosis complex in clinical samples from patients in Norway by ligase chain reaction. 7 Clin Microbiol 1997;35:3248-53.

17 Chan CM, Yuen KY, Chan KS, et al. Single-tube nested PCR in the diagnosis of tuberculosis. F Clin Pathol 1996;49: $290-4$.

18 Longo MC, Berninger MS, Hartley JL. Use of uracil DNA glycosylase to control carry over contamination in polymerase chain reactions. Gene 1990:93:125-8. 